\title{
EXTINCTION CURVES FOR GRAPHITE-SILICATE GRAIN MIXTURES
}

\author{
N. C. WICKR A M ASINGHE
}

Institute of Theoretical Astronomy, University of Cambridge, Cambridge, England

\begin{abstract}
Mixtures of graphite particles ejected from carbon stars and silicates from oxygen-rich giants are capable of producing excellent fits to the observed interstellar extinction curve. The fits extend over the entire wavelength range of the observations, and include a hump at $\sim 2200 \AA$ in the ultraviolet due to the graphite components of the mixtures. The agreement with the observed extinction curve remains good if the small silicate particles acquire mantles of either ice or solid $\mathbf{H}_{2}$. If no mantles are present around the silicate grains comparable mass densities of graphite and silicates are indicated; if mantles of either ice or solid $\mathrm{H}_{2}$ are present the mass density of silicates may be an order of magnitude below that of graphite in the interstellar medium.
\end{abstract}

\section{Introduction}

The recent discovery of a broad emission feature at $\sim 10 \mu$ in the spectra of several oxygen-rich giants has led to the belief that silicate particles are produced in these stars and are ejected into the interstellar medium (Woolf and Ney, 1969; Knacke et al., 1969; Ney and Allen, 1969; Stein and Gillett, 1969; Gilman, 1969). The carbon stars, on the other hand, are expected to produce graphite particles which are also ejected into the interstellar medium (Hoyle and Wickramasinghe, 1962; Wickramasinghe, 1967). Interstellar grains may thus be regarded as a mixture of at least two distinct, highly refractory components - graphite and silicates. The astrophysical consequences of such a grain mixture have already been discussed in an earlier paper (Hoyle and Wickramasinghe, 1969). We present here in more detail a discussion of the optical properties of such mixtures with particular reference to the interstellar extinction curve in the rocket ultraviolet.

\section{Graphite Particles}

The interstellar extinction curve is now known over a wide waveband $\sim 0.3 \leqslant \lambda^{-1} \leqslant$ $\leqslant 9 \mu^{-1}$, and the requirement of matching this entire curve might be expected to impose stringent constraints on theoretical models of the grains. The original rocket ultraviolet observation of Stecher (1965) besides showing a generally rising extinction curve for $\lambda<3000 \AA$ also indicated a rather conspicuous hump at $\sim 2200 \AA$. This hump which has recently been confirmed independently by Stecher (1969) and by Bless and Savage (1969) coincides with a similar hump present in the calculated extinction curves for graphite particles (Wickramasinghe and Guillaume, 1965; Stecher and Donn, 1965). The graphite feature arises due to the transition of $\pi$-electrons to the conduction band of graphite. The astronomical observations could thus be interpreted as implying the presence of a graphite component to the grains causing the extinction of starlight. 


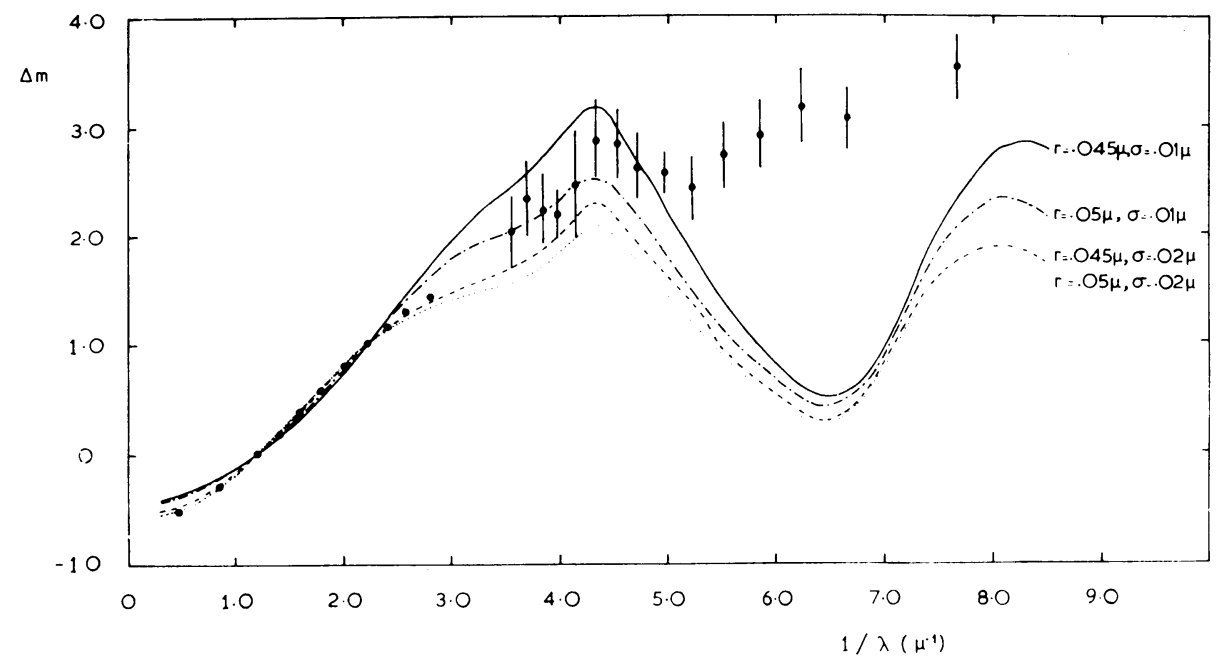

Fig. 1. Normalised extinction curves for size distributions of graphite particles defined by Equation (1) with $\bar{r}, \sigma$ values characterising the several curves denoting the mean radii and dispersions. Normalisation is to $\Delta m=0$ at $\lambda^{-1}=1.22 \mu^{-1}, \Delta m=1$ at $\lambda^{-1}=2.22 \mu^{-1}$. The observational points are those of Johnson (1965) for Cygnus in the infrared, Nandy (1964) for Cygnus in the optical and of Stecher (1965), and Bless et al. (1968) in the ultraviolet. The observations are also normalised in the same manner as the theoretical curves.

We have calculated the extinction resulting from a size-distribution of graphite particles defined by

$$
n(a) \propto \exp \left[-\left(a-\bar{r}^{2}\right)^{2} /\left(2 \sigma^{2}\right)\right]
$$

for various values of $\bar{r}$ and $\sigma . n(a) \mathrm{d} a$ represents the number of grains per $\mathrm{cm}^{3}$ with radii between $a, a+\mathrm{d} a$ and the distribution is truncated to zero outside the range $\bar{r}-1.5 \sigma<a<\bar{r}+3.5 \sigma$. The optical constants of graphite were taken from the data of Taft and Phillipp (1965) and the normalization is chosen such that

$$
\begin{aligned}
& \Delta m=0 \text { at } \lambda^{-1}=1.22 \mu^{-1} \\
& \Delta m=1 \text { at } \lambda^{-1}=2.22 \mu^{-1} .
\end{aligned}
$$

Figure 1 shows normalized extinction curves for several combinations of $\bar{r}, \sigma$ compared with the observational points of Johnson (1965) in the infrared for Cygnus, of Nandy (1964) in the optical for Cygnus, and of Stecher (1965) and Bless et al. (1968) in the rocket ultraviolet. All the curves in Figure 1 exhibit a pronounced hump at $\lambda \approx 2200 \AA$. The best over-all agreement with the observations in the infrared, optical and near ultraviolet occurs for the case $\bar{r}=0.045-0.05 \mu, \sigma \simeq 0.02 \mu$, but it seems unavoidable that the theoretical curves for pure graphite flakes diverge significantly from the observations for $\lambda \lesssim 2000 \AA$. Graphite core-ice mantle grains are capable of producing a general rise in the extinction curve further into the ultraviolet, but the graphite hump at $\sim 2200 \AA$ is then completely washed out (Wickramasinghe, 1967). While graphite core-solid $\mathrm{H}_{2}$ grains could retain this hump, 
the formation and stability of such mantles may yet be open to question (Wickramasinghe and Nandy, 1968; Wickramasinghe and Krishna Swamy, 1969).

\section{Graphite-Silicate Mixtures}

We discuss here the possibility that graphite and silicate particles may exist as separate entities in the interstellar medium. Consider a size-distribution of graphite particles defined by (1) mixed with silicate particles of radii $r_{\text {sil }}$. The relative proportions of graphite to silicates is specified by a parameter $x$ given by

$$
x=\frac{\text { extinction by graphite component at } 4500 \AA}{\text { extinction by silicate component at } 4500 \AA} \text {. }
$$

We carried out extinction calculations with various values of the size parameters $\bar{r}_{\mathrm{gr}}, \sigma, r_{\mathrm{sil}}$ and with various values of $x$. The complex refractive index of the silicates was taken to be $m=1.66$ over the entire wavelength range of interest. For $r_{\text {sil }}$ in the range $0.07-0.09 \mu$ and $\bar{r}_{\mathrm{gr}} \sim 0.05-0.06 \mu$ the best fits emerged for $x \simeq 6$ - that is, for 6 times as much extinction at $4500 \AA$ arising from the graphite component as from the silicate component (see also Hoyle and Wickramasinghe, 1969). The normalised extinction curves for

$$
\bar{r}_{\mathrm{gr}}=0.05,0.06 \mu, \sigma=0.02 \mu ; \quad r_{\mathrm{sil}}=0.07,0.08 \mu,
$$

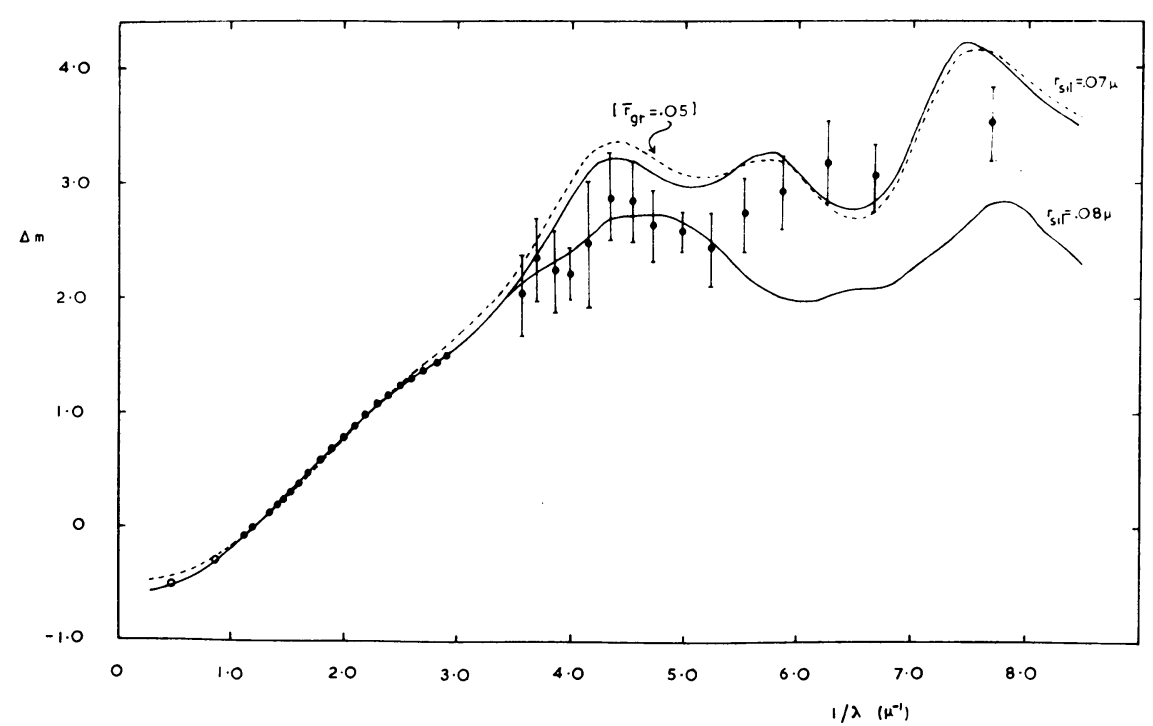

Fig. 2. Solid curves are the normalised extinction for size distributions of graphite particles defined by Equation (1) characterised by $\bar{r}=0.06 \mu, \sigma=0.02 \mu$ mixed together with silicate particles of radii $r_{\mathrm{sil}}=0.07 \mu$ and $0.08 \mu$. The particle types are mixed so that 6 times as much extinction at $4500 \AA$ arises from the graphite as from the silicate. The dashed curve is for the case $\bar{r}=0.05 \mu$, $\sigma=0.02 \mu$ and $r_{\text {sil }}=0.07 \mu$ with $x=6$. The observational points and the normalisation are the same as in Figure 1. 
with $x=6$ are plotted in Figure 2. The agreement between the theoretical curves in Figure 2 and the observational points (the same as Figure 1) is seen to be quite satisfactory. The graphite hump at $\sim 2200 \AA$ is well reproduced; the otrer humps in the far ultraviolet may be expected to be smoothed out by a distribution of sizes for $r_{\text {sil }}$.

If $n_{\mathrm{gr}}$ and $n_{\mathrm{sil}}$ are the number densities of the graphite and silicate components of the mixture the above arguments of a fit to the extinction with $x=6$ would imply that

$$
\begin{aligned}
& Q_{\text {ext }}(\text { graphite }) \pi r_{\mathrm{gr}}^{2} n_{\mathrm{gr}} \\
& Q_{\text {ext }}(\text { silicate }) \pi r_{\text {sil }}^{2} n_{\text {sil }}
\end{aligned}
$$

for extinction efficiencies evaluated at $4500 \AA$. With the adopted radii $\bar{r}_{\mathrm{gr}} \simeq 0.06 \mu$, $r_{\text {sil }} \simeq 0.07 \mu$ and the computed values $Q_{\text {ext }}($ graphite $)=2.7, Q_{\text {ext }}$ (silicate) $=0.37$ it follows from (4) that

$$
n_{\mathrm{gr}} \sim n_{\mathrm{sil}} \text {. }
$$

Since graphite and silicates have comparable specific gravities it follows that comparable mass densities of these materials are required to explain the extinction.

\section{Accretion of Volatile Mantles on Silicates}

Graphite particles with radii $\sim 0.06 \mu$ absorb optical and ultraviolet radiation with efficiency factors close to unity. If the particles are considered to be relatively pure - without the presence of low frequency impurity oscillators - the temperature taken up by such grains in a typical interstellar radiation field may be estimated at $\sim 40 \mathrm{~K}$. Grains at these temperatures may not possess mantles of any type. Solid $\mathrm{H}_{2}$ mantles will not be maintained, and ice mantles could have difficulty in forming at these high temperatures (Williams, 1968).

Silicate particles, on the other hand, would tend to take up lower temperatures due to their very low absorptivity $\left(Q<10^{-3}\right)$ at optical and near ultraviolet wavelengths. If their impurity induced low frequency conductivity exceeds the optical conductivity it is possible the grains do not take up a temperature significantly higher than $2.7 \mathrm{~K}$ (Hoyle and Wickramasinghe, 1969); the condensation of solid $\mathrm{H}_{2}$ mantles may then be possible. In any case it seems highly likely that their temperatures will be less than $\sim 10 \mathrm{~K}$, so that the formation of ice mantles could occur. We consider next the extinction properties of graphite/silicate mixtures if (a) ice mantles form around the silicates, and (b) solid $\mathrm{H}_{2}$. mantles form around the silicate cores.

\section{Graphite Particle/Silicate Core-Ice Mantle Mixture}

As before we consider two component grain mixtures with the relative proportions of graphite grains and silicate core-ice mantle grains defined by the parameter

$$
x=\frac{\text { extinction by graphite component at } 4500 \AA}{\text { extinction by silicate core-mantle grains at } 4500 \AA} .
$$




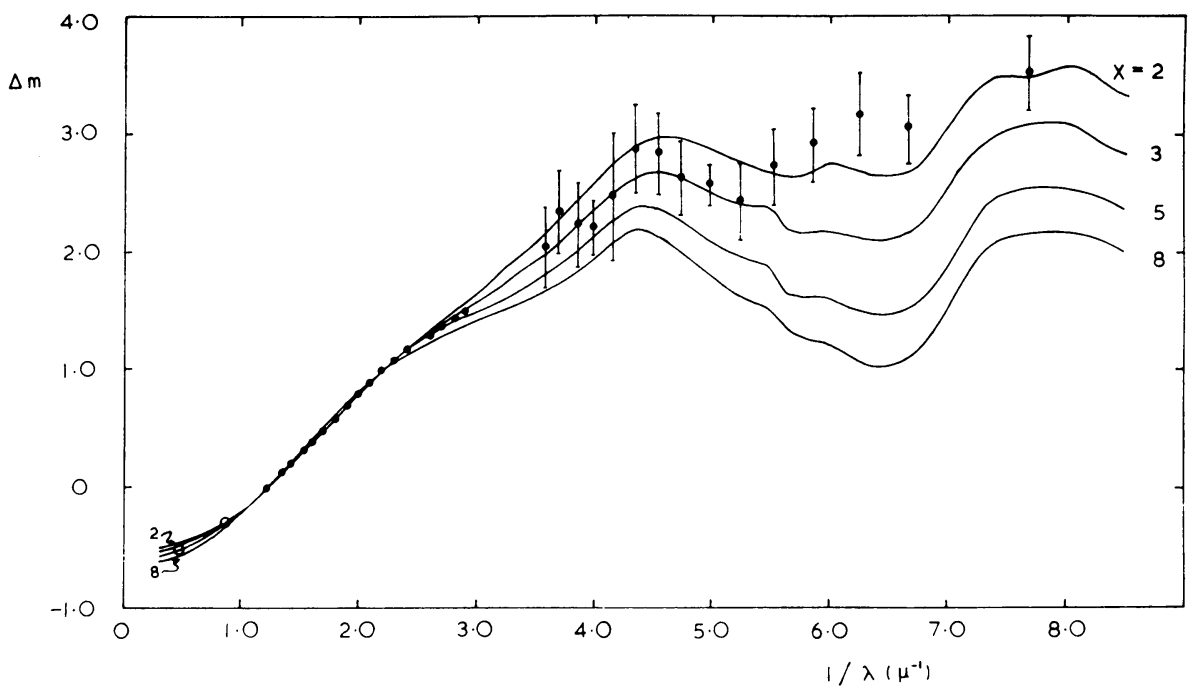

Fig. 3. Normalised extinction curves for a graphite size distribution given by (1) with $\bar{r}_{\mathrm{gr}}=0.06 \mu$, $\sigma=0.02 \mu$ mixed together with silicate core-ice mantle grains. The silicate core radius is $0.03 \mu$, the ice mantle radius is $0.14 \mu$ and the several curves $x=2-8$ represent mixtures in proportions defined

by Equation (5). The observational points and the normalization are the same as in Figure 1.

We adopt $m=1.33$ for the refractive index of ice at all wavelengths involved ${ }^{*}$ and consider a distribution of graphite particles given by (1) with $\bar{r}=0.06 \mu, \sigma=0.02 \mu$. The normalised extinction for the case $r_{\text {sil }}=0.03, r_{\text {ice }}=0.14 \mu$ with $x=2,3,5$ and 8 are plotted in Figure 3, together with the observational points. The best agreement is seen to occur for the case $x \cong 2$. As before the agreement includes the graphite hump at $2200 \AA$.

If $n_{\mathrm{gr}}, n_{\mathrm{sil}}$ represent the number densities of graphite particles and of silicate cores the fits obtained here imply

$$
\begin{aligned}
& Q_{\text {ext }}\left(\text { graphite) } \pi r_{\mathrm{gr}}^{2} n_{\mathrm{gr}}\right. \\
& Q_{\text {ext }}\left(\text { sil. core } / \mathrm{H}_{2} \mathrm{O}\right) \pi r_{\mathrm{H}_{2} \mathrm{O}}^{2} n_{\text {sil }} \simeq 2
\end{aligned}
$$

with the $Q_{\text {ext }}$ values computed at $4500 \AA$. For the appropriate values involved here it turns out that $n_{\mathrm{gr}} \sim n_{\mathrm{sil}}$; and since the assumed radii of the silicates is $\sim \frac{1}{2}$ that of the graphite it follows that the mass density of silicates is a factor $\sim 10$ lower than that of the graphite.

\section{STRENGTH OF THE 3.1 $\mu$-ICE BAND}

Attempts to detect the $3.1 \mu$ fundamental band of solid $\mathrm{H}_{2} \mathrm{O}$ in the spectra of highly reddened stars have yielded negative results on two successive occasions (Danielson

* The replacement of $m=1.33$ with the actual wavelength-dependent refractive index of $\mathrm{H}_{2} \mathrm{O}$-ice is likely to cause a slight lowering of the extinction curve for $i<1600 \AA$ in any given case. The general appearance of the curves including the 'hump' is likely to be unaltered, however. 


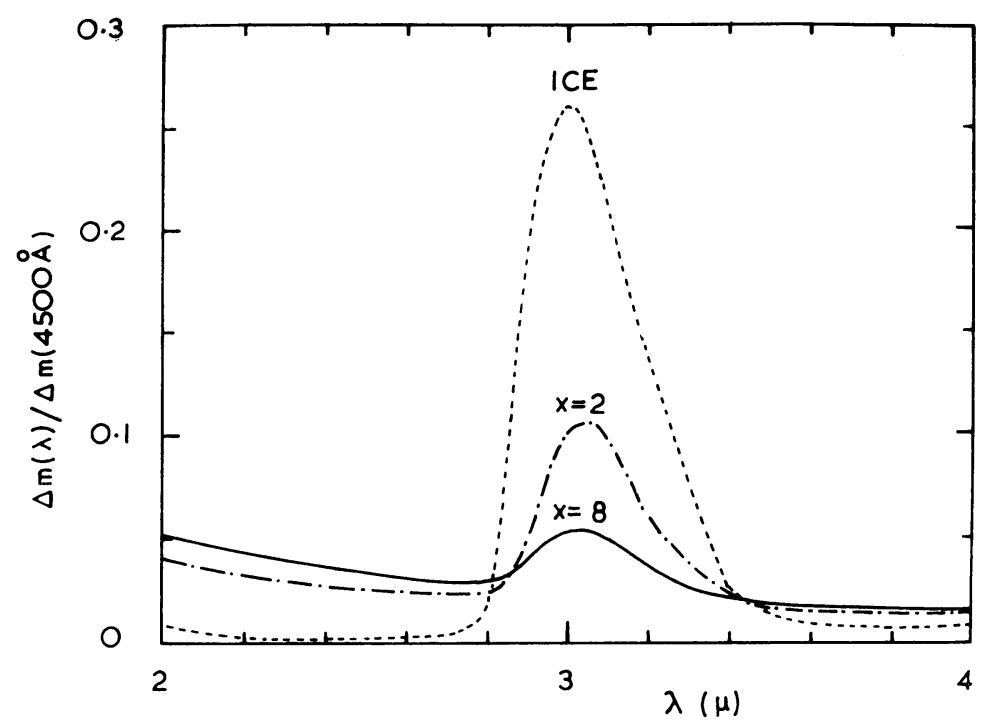

Fig. 4. The extinction near the $3.1 \mu$ ice-band per unit extinction at $4500 \AA$ for mixtures of silicate core-ice mantle grains and graphite particles with the same size parameters as in Figure 3 . The uppermost dashed curve is appropriate to an ice sphere of radius $0.14 \mu$, and the other curves $x \cdots 2,8$ correspond to 2 and 8 times as much extinction at $4500 \AA$ arising from the graphite component of the mixture as from the non-graphite component.

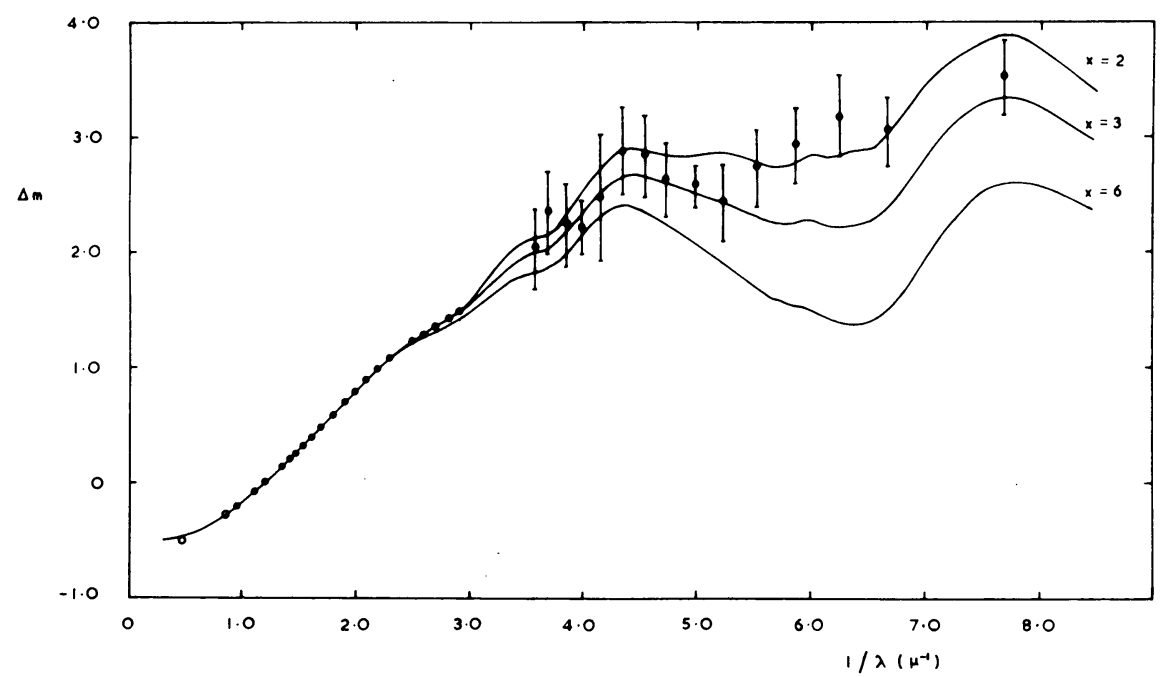

Fig. 5. Normalised extinction curves for a graphite size-distribution given by (1) with $\bar{r}_{\mathrm{gr}}=0.05 \mu$, $\sigma=0.02 \mu$ mixed with silicate core-solid $\mathrm{H}_{2}$ mantle grains. The radius of the silicate cores is $0.05 \mu$ and the solid $\mathrm{H}_{2}$ mantle radius is $0.35 \mu$. The several curves marked $x=2,3,6$ stand for mixtures with respectively $2,3,6$ times as much extinction at $4500 \AA$ arising from the graphite component as from the non-graphite component. The observational points and normalization are the same as in Figure 1. 
et al., 1965; Knacke et al., 1969). Although these results are yet to be regarded as somewhat preliminary, a quantity of obvious interest in any grain model involving $\mathrm{H}_{2} \mathrm{O}$-ice is the strength and shape of the $3.1 \mu$ ice band. For the silicate $-\mathrm{H}_{2} \mathrm{O} /$ graphite mixtures discussed above we have computed the quantity

$$
E(\lambda)=Q_{\mathrm{ext}}(\lambda) / Q_{\mathrm{ext}}(5000 \AA)
$$

within and in the vicinity of this band. To calculate $Q_{\text {ext }}(\lambda)$ in the far infrared we replaced the composite silicate-ice grains by pure ice spheres of the same outer radius. In Figure 4 we have plotted the normalised extinction $E(\lambda)$ for mixtures of graphite particles characterised by $\bar{r}=0.06 \mu, \sigma=0.02 \mu$ and silicate $-\mathrm{H}_{2} \mathrm{O}$ grains of inner radius $0.03 \mu$ and outer radius $0.14 \mu$. The relative proportions of the two separate components are as before defined by the parameter $x$ (from (5)). The observational limits on $E(3.1 \mu)$ set by the observations of Knacke et al. (1969) would seem to indicate that $x>2$ in the direstion of HD 183143 and $x>8$ in the directions of Vl Cyg. No 12 and CIT 11. The possibility that much of the reddening in these objects occurs nearby to the stars may not be completely ruled out at the present moment, so that these values of $x$ may well turn out to be untypical of the general interstellar medium.

\section{Graphite Particle/Silicate Core-Solid $\mathrm{H}_{2}$ Mantle Mixture}

Finally we present extinction curves for the case when solid $\mathrm{H}_{2}$ mantles may be present around the silicate particles in a mixture. Again we take a graphite particle size-distribution of the form (1) and prescribe the relative proportions of graphite and silicate core-mantle grains by the parameter $x$ defined by (5). For $\bar{r}=0.05 \mu$, $\sigma=0.02 \mu$ as the graphite parameters and $r_{\text {sil }}=0.05 \mu, r_{\mathrm{H}_{2}}=0.35 \mu$ the normalised extinction curves are given in Figure 5 for various values of $x$. The best agreement with the observational points is seen to occur for the case $x=2$. As in the previous cases it is seen that the graphite hump at $2200 \AA$ is well fitted. The mass density of silicates in the interstellar medium is again at least an order of magnitude below that of graphite.

\section{Acknowledgement}

I thank Professor F. Hoyle for several discussions.

\section{References}

Bless, R. C., Code, A. D., and Houck, T. E.: 1968, Astrophys. J. 153, 561.

Bless, R. C. and Savage, B. D.: 1969, This Symposium, p. 28.

Danielson, R. E., Woolf, N. J., and Gaustad, J. E.: 1965, Astrophys. J. 141, 116.

Gilman, R. C.: 1969, Astrophys. J. 155, L185.

Hoyle, F. and Wickramasinghe, N. C.: 1962, Monthly Notices Roy. Astron. Soc. 124, 417.

Hoyle, F. and Wickramasinghe, N. C.: 1969, Nature 223, 459.

Johnson, H. L.: 1965, Astrophys. J. 141, 923.

Knacke, R. F., Gaustad, J. E., Gillett, F. C., and Stein, W. A.: 1969, Astrophys. J. 155 , L189.

Nandy, K.: 1964, Publ. Roy. Observ. Edin. 3, No. 6.

Ney, E. P. and Allen, D. A.: 1969, Astrophys. J. 155, L193. 
Stecher, T. P.: 1965, Astrophys. J. 142, 1683.

Stecher, T. P.: 1969, This Symposium, p. 24. Astrophys. J. 157, L125.

Stecher, T. P. and Donn, B.: 1965, Astrophys. J. 142, 1681.

Stein, W. A. and Gillett, F. C.: 1969, Astrophys. J. 155, L197.

Taft, E. A. and Phillipp, H. R.: 1965, Phys. Rev. 138A, 197.

Wickramasinghe, N. C.: 1967, Interstellar Grains, Chapman and Hall, London.

Wickramasinghe, N. C. and Guillaume, C.: 1965, Nature 207, 366.

Wickramasinghe, N. C. and Krishna Swamy, K. S.: 1969, Monthly Notices Roy. Astron. Soc. 144, 41. Wickramasinghe, N. C. and Nandy, K.: 1968, Nature 219, 1347.

Williams, D. A.: 1968, Astrophys. J. 151, 935.

Woolf, N. J. and Ney, E. P.: 1969, Astrophys. J. 155, L181. 